\title{
CNC-LIKE INJECTION MACHINE APPLIED INTO SMD DEVICES WITH PASTE SOLDER TECHNIQUE
}

\author{
Marcos Vinicius GONÇALEZ ${ }^{1}$, JiLMAR PEREIRA SILVA ${ }^{1}$, LUPÉRCIO JUNIOR AVANÇO ${ }^{1}$, EdNO JunioR \\ APARECIDO $^{1}$, LUCIANO CÁSSIO LULIO $^{1,2}$.
}

\author{
1. Instituto de Ciências Exatas e Tecnologia, Universidade Paulista (ICET/UNIP) \\ Av. Juscelino Kubitschek de Oliveira, s/n, São José do Rio Preto, SP - Brasil \\ E-mail: marrcos_vinicius@yahoo.com.br
}

\author{
2. Núcleo de Ensino e Pesquisa em Automação e Simulação, Escola de Engenharia de São Carlos, Uni- \\ versidade de São Paulo (NEPAS/EESC/USP) \\ Av. Trabalhador São-carlense, $n^{\circ}$ 400, São Carlos, SP - Brasil \\ E-mail: lclulio@sc.usp.br
}

\begin{abstract}
This projects aims to develop a CNC injection machine (Computerized Numerical Control) equipped with 4 axis, X, $\mathrm{Y}, \mathrm{Z}$ and also the A axis, responsible for the linear fit of the injection of the weld. Its main function, is to perform automated solder of electronic components of SMD technology (Surface Mounting Device). The machine provides low cost construction components, because it is directed for easy acquisition benefits in small electronic industries and companies, which do not have sufficient financial resources to acquire dedicated industrial machines (high cost and complex operations). The concern during development is to present propositions technical and economically feasible for the manual welding process, because the automated welding ensures greater accuracy, high productivity, and reduction of errors. The welding is performed using resin solder paste sustainable Lead-Free(No Lead), with 96.5\%Snalloys, Ag 3.0\% Cu and 0.5\% (suitable material for use in SMD circuits), the injection resin core solder (flow), constituted by micro spheres which will be deposited on the PCI card, and covering the surfaces to be welded, the $\mathrm{Z}$ axis is equipped with a dispenser, that effects the dosage of the resin for the board surface solder.
\end{abstract}

Keywords_ CNC injection machine, Paste solder dispenser, Resin solder, Automated soldering.

Resumo- Este projeto tem como objetivo o desenvolvimento de uma máquina de injeção de solda CNC, equipada com 4 eixos (X, Y, Z, a também o eixo A, responsável pela inserção de pasta. Sua função principal é prover soldagem automatizada em componentes eletrônicos SMD. A máquina provê também a construção com componentes de baixo custo, direcionada a indústrias e companhias eletrônicas de médio porte. O propósito acerca do projeto consiste em apresentar técnicas mais eficientes de soldagem, e tornar o processo de solda mais dinâmico, uma vez que sua automação admite maior precisão, maior produtividade, e redução de erros. A soldagem é realizada usando resina de solda em pasta do tipo Lead-Free, com 96,5\% de liga de estanho, Prata $3,0 \%$ e Cobre $0,5 \%$.

Palavras-chave— Máquina de injeção CNC, Dispensor de pasta de solda, Resina de solda, Solda automatizada.

\section{Introdução}

O presente trabalho propõe o desenvolvimento de uma máquina CNC (Controle Numérico Computadorizado), cuja função é efetuar a injeção de solda em PCI (Placa de Circuito Impresso) com componentes SMD (Dispositivos de Montagem em Superfície), permitindo injeção de pasta de solda de maneira repetitiva e proporcional aos dispositivos com dimensões reduzidas, garantindo a qualidade do processo. Quando comparado com o processo manual, a solda automatizada em circuitos SMDs, garante maior precisão e controle de qualidade, através do ajuste da dosagem da pasta de solda

\section{Materiais e métodos}

O chassi da máquina de solda em pasta é formado por cinco guias lineares de gavetas, cuja função, será permitir o deslocamento do dispensador ao longo do eixo $\mathrm{x}$, y e $\mathrm{z}$, o deslocamento do eixo $\mathrm{y}$, será feito com a ajuda de um motor de passo acoplado a um fuso trapezoidal, este fuso será acoplado na parte interna da estrutura. O chassi apresenta tampas laterais que podem ser removidas para efeito de manutenção, tanto no motor de passo como em qualquer componente integrante da máquina que necessite de manutenção, pensando nisso foi desenvolvido o designer prático, para facilitar a realização de reparos na placa do circuito ou caso os componentes eletrônicos tenha que ser verificados.

\subsection{Eixos de atuação na máquina}

A máquina será do tipo PPP, com coordenadas cartesianas nos três eixos prismáticos e um quarto eixo rotacional (que não influenciará nos cálculos de cinemática direta), esse quarto eixo será responsável pela dosagem da pasta de solda. A máquina poderá mover em linhas retas, com deslocamentos horizontais e verticais, e além dos eixos prismáticos há um eixo A, que efetuará movimento rotacional em torno do seu próprio eixo. As coordenadas cartesianas especificam um ponto no espaço em função de suas coordenadas $\mathrm{X}, \mathrm{Y}$ e Z. 


\subsection{Pasta de solda}

A pasta de solda consiste em esferas de solda e fluxo líquido. A pasta de solda é especificada pela liga do metal e o tamanho das esferas. Ligas típicas contêm uma combinação de chumbo, estanho, prata, bismuto, índio, cobre e outras ligas. As ligas são escolhidas pelas suas propriedades físicas e químicas para uma aplicação específica, podendo afetar no rendimento da aplicação.

\subsection{Volumes de pasta de solda}

A Pasta de solda deve ser depositada nas ilhas da placa PCI moderadamente, o excesso ou a falta de volume acarretará falhas ao inserir os componentes. Dispensar uma quantidade muito pequena, muito grande, ou variando o volume sem ter conhecimento da área a ser preenchida pode causar problemas. Uma boa regra é dispensar um volume entre $1 / 4$ a $1 / 2$ do diâmetro da área a ser preenchida, ou seja, para uma área cujo diâmetro é de $1 \mathrm{~mm}^{2}$, o ideal é dispensar um volume de $0,25 \mathrm{~mm}^{3}$ a $0,5 \mathrm{~mm}^{3}$. Sendo dispensada por um sistema de válvula controlada por pressão, dotado de uma rosca helicoidal.

\subsection{Itens da válvula dispensadora}

1. Suporte para acoplamento da válvula dispensadora na estrutura da máquina, fixada por dois parafusos, com o intuito de manter a válvula fixa, evitando eventuais folgas que levaria a um desalinhamento perpendicular da ponta em relação à placa de circuito impresso.

2. Cabos de alimentação DC do servo motor que efetuará a rotação do eixo da rosca, conectados ao controlador da válvula e diferenciado pela cor Branca (+) e Marron (-).

3. Adaptador para mangueira acoplado na saída do reservatório de pasta de solda.

4. Pinça de fixação do reservatório de pasta de solda, cujo aperto da borboleta da pinça não deve ser demasiado, pois ocasionará pressão excessiva da garra no adaptador da mangueira, podendo danificalo.

5. Compreende de uma Conexão "T" com encaixe para o parafuso dosador de solda, a posição do distribuidor dosador, deve ser posicionado com o encaixe da entrada da mangueira voltado para a parte frontal da válvula.

6. Mangueira de alimentação de solda, ligada do adaptador ao conjunto " $T$ ", o comprimento útil da mangueira deve ser de $80 \mathrm{~mm}$ e diâmetro de $3 \mathrm{~mm}$ ou $1 / 8$ ".

7. Tampa do reservatório de pasta de solda, cuja finalidade é vedar todo bocal do reservatório impedindo a saída de ar comprimido, criando uma câmara pressurizada de no máximo 2,1 bar dentro do reservatório. A falha na vedação do bocal permitindo fuga de pressão poderá ocasionar problemas em manter a solda homogênea causando volume desproporcional durante o trabalho.
8. A escolha da agulha de injeção de solda deve ser revista para cada volume ou processo em que será dispensado, existem vários modelos e tamanhos de pontas.

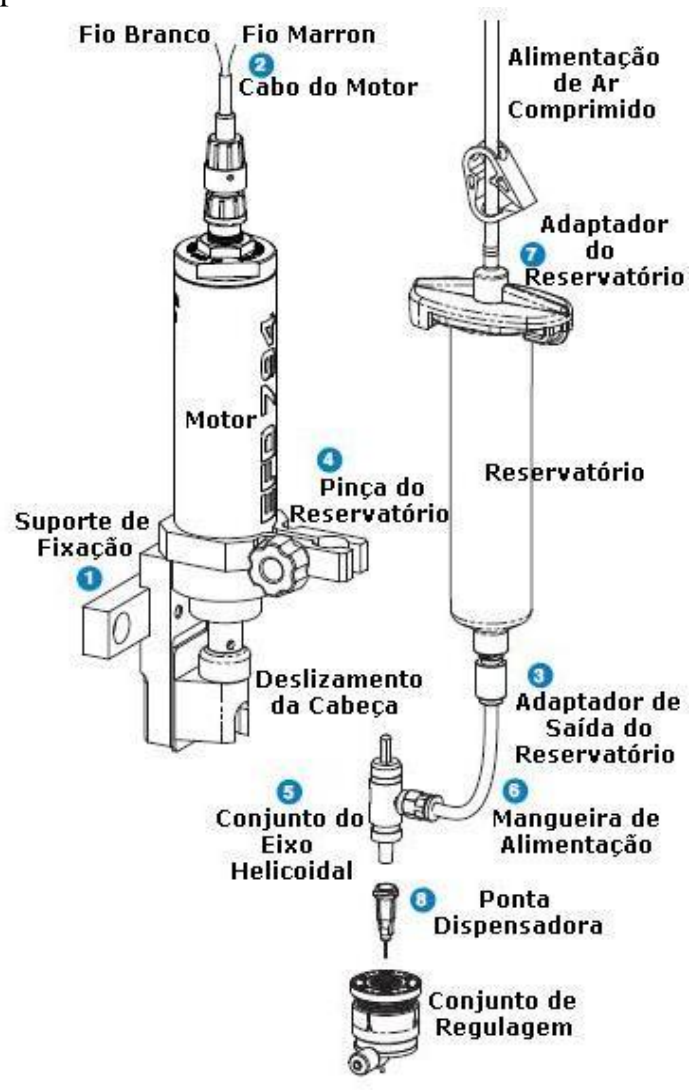

Figura 1. Componentes da válvula dispensadora (Modelo 794-SB).

Na Figura 2, uma Pressão de entrada de ar de até 30 psi (2,1 bar) é aplicada ao reservatório de material (1), forçando o fluido para dentro do caminho da rosca. À medida que a rosca sem-fim inicia um movimento rotacional, o fluído move-se ao longo do eixo helicoidal (2) e é forçado para fora através do orifício de distribuição (ponta da agulha). O controlador de válvula regula a pressão do reservatório, garantindo que não faltará pressão suficiente para manter a válvula abastecida com o líquido, sem forçar além da rosca sem-fim.

A duração do tempo de distribuição é controlada através do Controlador Válvula de Deslocamento Valve Mate ${ }^{\mathrm{TM}}$ Série 7094, até que o tamanho do depósito necessário seja estabelecido, e a altura que é depositada as gotas ocorre no inicio de cada ciclo até o seu final, dependendo da viscosidade do fluído a pressão poderá ser inferior a 2,1 bar. Na Figura 2, observa-se como ocorre o processo no interior da válvula. 


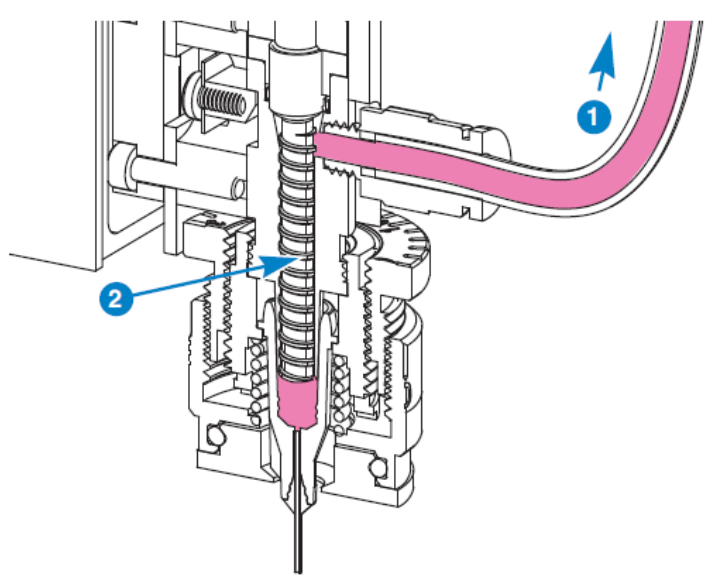

Figura 2. Teoria de aplicação (Modelo 794-SB).

Na Figura 3, é possível observar as dimensões da válvula de 237,5 mm de comprimento x 31,8 $\mathrm{mm}$ de diâmetro, o peso da válvula quando o reservatório está no seu limite máximo $(30 \mathrm{cc})$ é de $0,544 \mathrm{Kg}$, abastecida por ar comprimido de 0 a 2,1 bar limpo, seco e filtrado, sendo o parafuso de aço inoxidável resistente do tipo 440C. A tensão de entrada será de 24 VDC, tensão que pode ser controlada pelo controlador da Válvula de Deslocamento ValveMate.

A válvula não foi projetada, e sim subsidiada pela empresa EFD, portanto considera que a geometria da válvula foi dimensionada pela empresa mentora, visando uma fácil permutação entre agulhas, manutenção e troca reparos de peças quando necessário, tornando o projeto da válvula simples, porém preciso.

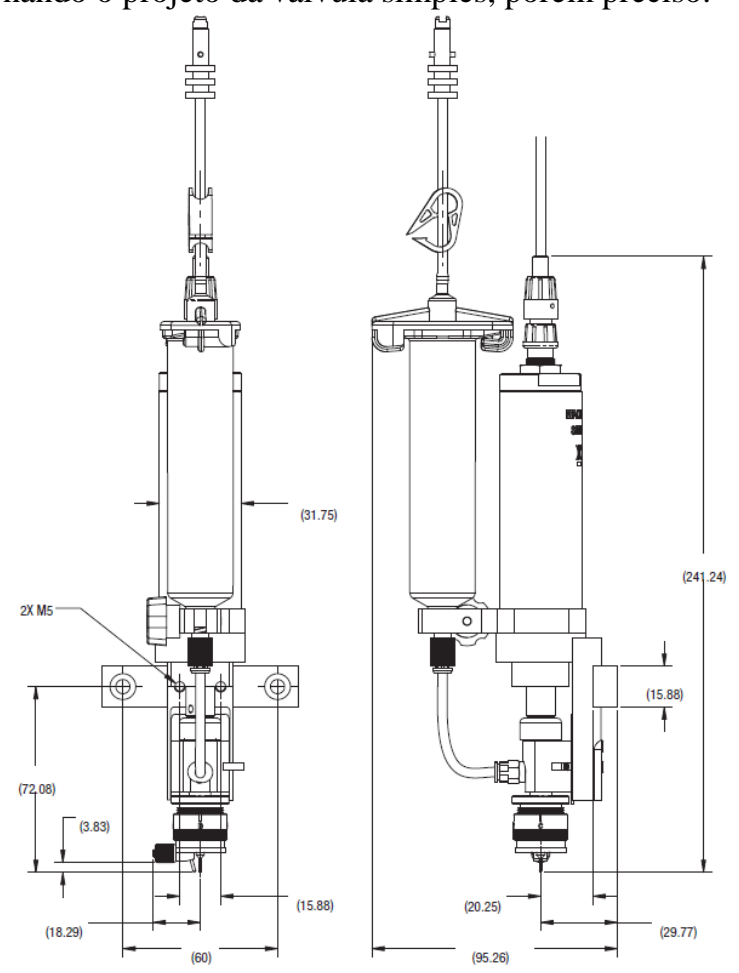

Figura 3. Dimensionamento da válvula dispensadora.

\subsection{Itens da válvula dispensadora}

Com base na cinemática direta, obtém-se um ponto no espaço, aplicando a modelagem matemática ao robô manipulador PPP. Na Figura 05, observam-se as configurações cartesianas para cada junta, sendo o eixo X, Y e Z prismáticos, sendo calculado a partir da tabela de Denavit-Hartenberg.

A máquina possui além dos três eixos prismáticos, uma rotação do servo motor para o eixo A acoplado ao eixo Z, com a finalidade de dosar a solda. Esse eixo, não será levado em consideração para fins de cálculos da modelagem cinemática, pois não influência na posição dos demais elos.

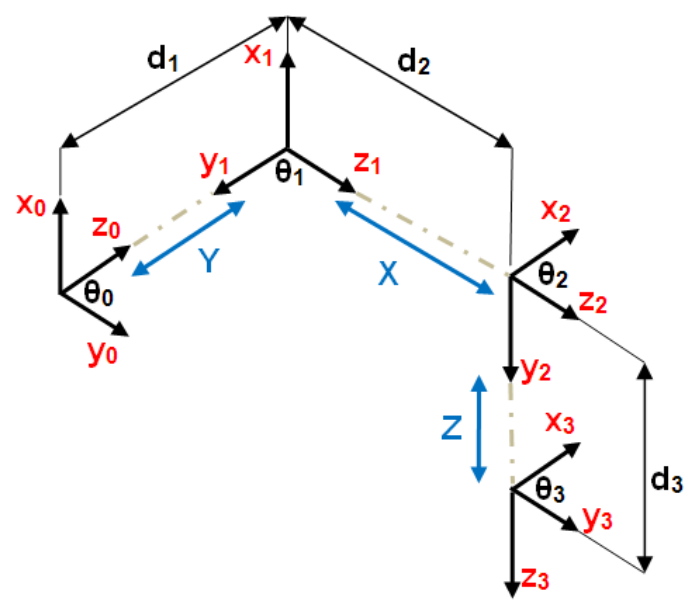

Figura 4. Orientação cinemática da máquina injetora.

\subsection{Obtenção das matrizes}

Através das matrizes de rotação, é possível determinar as rotações da origem até o ponto estimado.

$$
\begin{aligned}
R_{0}^{1}=\left[\begin{array}{lll}
i_{1} i_{0} j_{1} i_{0} & k_{1} i_{0} \\
i_{1} j_{0} j_{1} j_{0} & k_{1} j_{0} \\
i_{1} k_{0} j_{1} k_{0} k_{1} k_{0}
\end{array}\right] & =\left[\begin{array}{cc}
\cos 0^{0} & \cos 90^{0} \cos 90^{0} \\
\cos 90^{0} & \cos 0^{0} \cos 90^{\circ} \\
\cos 90^{0} \cos 90^{0} \cos 0^{0}
\end{array}\right]= \\
& =\left[\begin{array}{l}
100 \\
010 \\
001
\end{array}\right]
\end{aligned}
$$

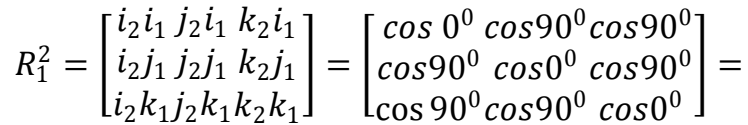

$$
\begin{aligned}
& =\left[\begin{array}{l}
100 \\
010 \\
001
\end{array}\right]
\end{aligned}
$$

$$
\begin{aligned}
& R_{2}^{3}=\left[\begin{array}{lll}
i_{3} i_{2} j_{3} i_{2} & k_{3} i_{2} \\
i_{3} j_{2} j_{3} j_{2} & k_{3} j_{2} \\
i_{3} k_{2} j_{3} k_{2} k_{3} k_{2}
\end{array}\right]=\left[\begin{array}{cc}
\cos 0^{0} & \cos 90^{0} \cos 90^{0} \\
\cos 90^{0} & \cos 0^{0} \cos 90^{0} \\
\cos 90^{0} \cos 90^{0} & \cos 0^{0}
\end{array}\right]= \\
& =\left[\begin{array}{l}
100 \\
010 \\
001
\end{array}\right]
\end{aligned}
$$

Nas equações 04, 05 e 06, são calculadas as matrizes de translação do manipulador. 


$$
\begin{gathered}
\mathrm{P}_{0}^{1}=\left[\begin{array}{l}
\mathrm{a}_{1} \cos \theta_{1} \\
\mathrm{a}_{1} \operatorname{sen} \theta_{1} \\
\mathrm{~d}_{1}
\end{array}\right]=\left[\begin{array}{c}
0 \\
0 \\
250
\end{array}\right] \\
\mathrm{P}_{1}^{2}=\left[\begin{array}{c}
\mathrm{a}_{2} \cos \theta_{2} \\
\mathrm{a}_{2} \operatorname{sen} \theta_{2} \\
\mathrm{~d}_{2}
\end{array}\right]=\left[\begin{array}{c}
0 \\
0 \\
250
\end{array}\right] \\
\mathrm{P}_{2}^{3}=\left[\begin{array}{c}
\mathrm{a}_{3} \cos \theta_{3} \\
\mathrm{a}_{3} \operatorname{sen} \theta_{3} \\
\mathrm{~d}_{3}
\end{array}\right]=\left[\begin{array}{c}
0 \\
0 \\
50
\end{array}\right]
\end{gathered}
$$

Os dados a seguir, referem-se as Matrizes Homogêneas.

$$
\begin{aligned}
\mathrm{H}_{0}^{1} & =\left[\begin{array}{cccc}
1 & 0 & 0 & 0 \\
0 & 1 & 0 & 0 \\
0 & 0 & 1 & 250 \\
0 & 0 & 0 & 1
\end{array}\right] \\
\mathrm{H}_{1}^{2} & =\left[\begin{array}{cccc}
1 & 0 & 0 & 0 \\
0 & 1 & 0 & 0 \\
0 & 0 & 1 & 250 \\
0 & 0 & 0 & 1
\end{array}\right] \\
\mathrm{H}_{2}^{3} & =\left[\begin{array}{lllc}
1 & 0 & 0 & 0 \\
0 & 1 & 0 & 0 \\
0 & 0 & 1 & 50 \\
0 & 0 & 0 & 1
\end{array}\right]
\end{aligned}
$$

Para encontrar a matriz de transformação homogênea geral do sistema, é efetuado o produto de todas as matrizes.

$$
\begin{gathered}
\mathrm{T}_{3}^{0}=\mathrm{H}_{0}^{1} \cdot \mathrm{H}_{1}^{2} \cdot \mathrm{H}_{2}^{3} \\
\mathrm{~T}_{0}^{3}=\left[\begin{array}{cccc}
\mathrm{r}_{\mathrm{x}}^{1} & \mathrm{r}_{\mathrm{x}}^{2} & \mathrm{r}_{\mathrm{x}}^{3} & 0 \\
\mathrm{r}_{\mathrm{y}}^{1} & \mathrm{r}_{\mathrm{y}}^{2} & \mathrm{r}_{\mathrm{y}}^{3} & 0 \\
\mathrm{r}_{\mathrm{z}}^{1} & \mathrm{r}_{\mathrm{z}}^{2} & \mathrm{r}_{\mathrm{z}}^{3} & 550 \\
0 & 0 & 0 & 1
\end{array}\right]
\end{gathered}
$$

\subsection{Aplicação da pasta de solda}

Para demonstrar o processo de injeção da pasta de solda e precisão da máquina, foi utilizado um modelo de cartão com diversos pontos em forma de círculos, com diâmetros especificados para cada agulha. A máquina deverá posicionar o bico dosador e dispensar a solda até preencher o diâmetro dos pontos. Para a execução da tarefa, o Controlador Válvula de Deslocamento foi regulado para exercer a pressão de 0,5 bar no reservatório de pasta de solda, essa pressão será contínua no pistão do reservatório com a finalidade de garantir que a pasta de solda ao ser comprimida pelo pistão, preencha totalmente a rosca helicoidal da válvula, garantindo assim uma solda homogênea, isenta de bolha de ar no interior da válvula. Após os ensaios de verificação da precisão da máquina, será efetuado o ensaio de injeção de solda na PCI, com o intuito de simular a real aplicação do projeto. O diâmetro da ilha da PCI é de $1 \mathrm{~mm}$, portanto será utilizada a agulha em tom de Azul, por apre- sentar diâmetro externo da agulha o mais próximo do diâmetro da ilha, para depósito da pasta de solda.

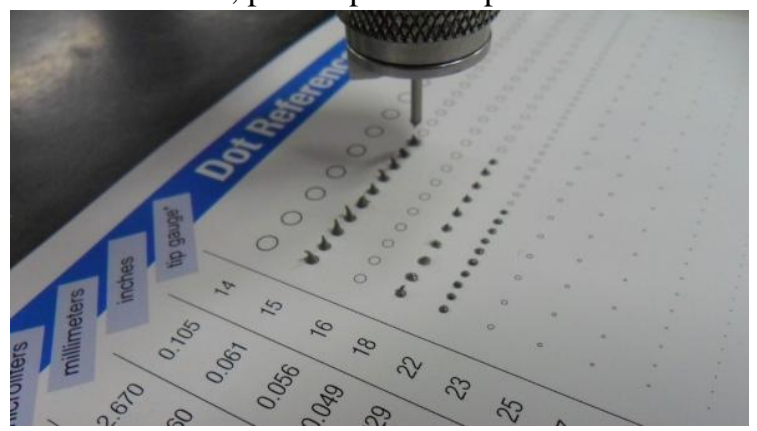

Figura 5. Visual da soldagem após teste com agulha azul.

Foi efetuado o teste na placa PCI, simulando a injeção da pasta de solda em uma área de diâmetro igual a $1 \mathrm{~mm}$. Portanto será utilizada a agulha em tom de Azul, por apresentar diâmetro externo da agulha o mais próximo do diâmetro da ilha, para depósito da pasta de solda. Os procedimentos adotados para deposição da solda na placa PCI foi, regulagem da altura entre a PCI e a ponta da agulha, de $2 \mathrm{~mm}$, com Tensão do Controlador da Válvula em 24 VDC e o tempo programado de rotação do eixo para cada ponto de descida foi de $250 \mathrm{~ms}$. A Figura 07 (a) é possível observar a máquina posicionada no ponto de referência zero, localizado no canto da PCI.

A PCI possui uma dimensão de 30 x 20 em milímetros, apresentando um total de 36 ilhas, foi injetado, portanto 36 pontos de solda ao total na placa, 10 ilhas com diâmetro $0,5 \mathrm{~mm}$ maior que as demais que possuem $1 \mathrm{~mm}$, na qual foi necessário aplicar um tempo maior de rotação do eixo. $\mathrm{O}$ tempo do eixo $\mathrm{z}$ foi programado $250 \mathrm{~ms}$ para os pontos com diâmetro de 0,5 $\mathrm{mm}$ e $320 \mathrm{~ms}$ para ilhas maiores.

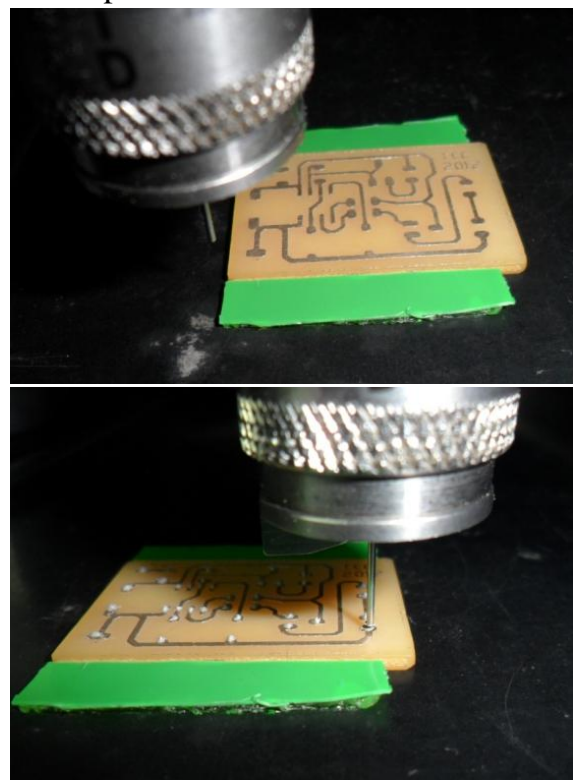

Figura 6. (a) Referência fim de curso; (b) PCI após receber os pontos de solda. 


\section{Conclusão}

O objetivo principal da máquina injetora de solda em pasta para SMD foi alcançado. A máquina foi projetada com o intuito de garantir uma soldagem de precisão, executando um processo de deposição de solda em pasta através de gotas dispensadas ainda pouco conhecida no Brasil. O estudo sobre as características da pasta de solda foi muito interessante, para o conhecimento de novas pastas com características sustentáveis, as pastas Lead-Free que em breve substituirão as convencionais e mais usuais pastas de Chumbo/Estanho $(\mathrm{Pb} / \mathrm{Sn})$, que apresentam elevado grau de risco, por serem cancerígenas, dado que muitas pessoas desconhecem.

Executar soldagem em placas PCI requer conhecimento, pois o processo contém detalhes minuciosos que devem ser levados em consideração. Quando aplicado nas indústrias, o processo tende ser bem aceito, pois o processo manual desprende um longo tempo do operador para efetuar tarefas de soldagem, e na maioria das vezes o resultado final não garante um acabamento de qualidade quando comparado a processos automatizados. O processo de deposição de pasta de solda desenvolvido neste projeto garante confiabilidade e qualidade acima de tudo e uma perfeita relação entre custo/beneficio. A soldagem pode ser feita sem a presença do operador, apenas com o layout configurado na tela da máquina, é possível percorrer e depositar automaticamente todos os pontos desejados de uma PCI, sendo possível trabalhar com pacotes BGA, levando em consideração os resultados e precisão alcançada nos testes efetuados. Os depósitos de pasta de solda que eram praticamente manuais poderão ser automatizados, utilizando os recursos semelhantes ao apresentado, e garantindo uma melhor qualidade do produto final.

\section{Agradecimentos}

Os autores agradecem ao ICET/UNIP, campus São José do Rio Preto, SP, pelo apoio e auxílio nos laboratórios técnicos durante o desenvolvimento do projeto.

\section{Referências Bibliográficas}

EFD - Engineered Fluid Dispensing, Electronic PDF files of EFD manuals are also available at, Sales and service of Nordson EFD dispensing systems are available worldwide. Disponível em: $<$ http://www.nordsonefd.com>. Acesso em: Junho, 2012.

LEWIS, A., Formulation Considerations for Automated Dispensing of Lead Free Solder Paste. Acesso em: Outubro de 2012.

WEDEKIN, S. "Micro-dispensing Comes of Age," in SMT Magazine, April 2001.
AGOSTINI, S. L. "Adesivo", in Tutoriais técnicos SMD, 04/2012. Disponível em: <smd-online.com>. Acesso em: Abril de 2012.

MANKO, Howard H. Soldering handbook for printed circuits and surface mounting. New York: Van Nostrand Reinhold, 1986. 430 p. ISBN 0-442-26423-2. 